\title{
Template-Free Synthesis of Chemically Asymmetric Silica Nanotubes for Selective Cargo Loading and Sustained Drug Release
}

\author{
Chao Deng, ${ }^{\dagger}$ Qian Zhang, ${ }^{\dagger}$ Cong Fu, ${ }^{\dagger}$ Fangzhou Zhou, ${ }^{\dagger}$ Wuli Yang,,${ }^{\ddagger}$ Deliang Yi, ${ }^{\dagger}$ Xingdong Wang, ${ }^{\S}$ Yi \\ Tang, ${ }^{\dagger}$ Frank Caruso, ${ }^{\mathbb{T}}$ and Yajun Wang ${ }^{* \dagger}$
}

'Department of Chemistry and Shanghai Key Laboratory of Molecular Catalysis and Innovative Materials, Fudan University, Shanghai 200433, P. R. China.

${ }^{\ddagger}$ State Key Laboratory of Molecular Engineering of Polymers, Department of Macromolecular Science, Fudan University, Shanghai 200433, P. R. China.

${ }^{\S}$ CSIRO Manufacturing, Private Bag 10, Clayton South, Victoria 3169, Australia.

${ }^{\pi}$ ARC Centre of Excellence in Convergent Bio-Nano Science and Technology, and the Department of Chemical Engineering, The University of Melbourne, Parkville, Victoria 3010, Australia.

\begin{abstract}
SiO}_{2}$ nanotubes (NTs), with uniform diameter $(\sim 25 \mathrm{~nm})$ and tailored shell thickness $(1-5 \mathrm{~nm})$, and featuring chemically asymmetric surfaces (a silane-functionalized exterior surface and a negatively charged $\left(-\mathrm{Si}-\mathrm{O}^{-}\right)$interior surface) are prepared by a facile, template-free approach. Owing to their chemically asymmetric properties, positively charged cargoes can be selectively loaded with high content into the $\mathrm{SiO}_{2} \mathrm{NTs}$ via electrostatic interaction. With the mediation of polyamidoamine dendrimer preassembled in the NTs, different noble metal nanoparticles can be selectively deposited inside the NTs with high density and uniform size. Owing to their high specific surface area and elongated pore structure with lengths up to several micrometers, the $\mathrm{SiO}_{2}$ NTs exhibit a high loading capacity $(80 \mathrm{wt} \%)$ for positively charged therapeutic agents, which exhibit sustained cargo release properties on the order of several months if the NTs are assembled into a membrane structure.
\end{abstract}

\section{INTRODUCTION}

Nanoarchitectured silica materials have received widespread interest in a range of applications (e.g., catalysis, ${ }^{1-3}$ sensing, ${ }^{4-6}$ drug delivery ${ }^{7-12}$ and templating synthesis ${ }^{13-18}$ ) owing to their unique morphologies, high surface areas, tunable pore structures, easily modifiable surface properties and high biocompatibility. The past two decades have witnessed the rapid development of silica materials with diverse architectures, ranging from ordered mesoporous frameworks, ${ }^{9,19-22}$ nanopods, ${ }^{23}$ nanorods, ${ }^{24}$ nanowires (NWs), ${ }^{25}$ nanocages, ${ }^{26}$ nanobottles $^{27}$ to nanotubes (NTs). ${ }^{28-31}$ NTs are of particular interest in nanoreactor and drug delivery applications owing to their elongated structures and multiple functionalities as a core material, which provide further opportunities to design nanoreactors with desirable geometric morphologies (e.g., assemblies of NT membranes) and unique reaction features, ${ }^{32-35}$ as well as the ability to control the biodistribution and release profiles of therapeutic agents. ${ }^{15,36} \mathrm{SiO}_{2}$ NTs are typically prepared using either a hard template (e.g., nickel-hydrazine nanorod, ${ }^{30}$ anodic aluminum oxide membrane $28,37,38$ ) or a soft template (e.g., threadlike block polymer micelles ${ }^{29,39}$ ) as a porogen to generate the hollow channels. However, these preparation methods largely depend on the availability of suitable templates. Furthermore, multiple process steps and harsh conditions (e.g., aggressive chemical etching or calcination) are typically re- quired to remove the templates from the products to generate the hollow channels. ${ }^{28-30}$ These limitations largely affect the scale-up synthesis and often compromise the quality of the NTs produced. Therefore, developing a template-free technique that yields high quality $\mathrm{SiO}_{2}$ NTs is of particular importance, both for technological applications and fundamental research.

Herein, we introduce a facile, template-free method to synthesize uniformly sized $\mathrm{SiO}_{2}$ NTs via an emulsion linearmerging growth (ELMG) approach. To the best of our knowledge, this is the first report on the synthesis of $\mathrm{SiO}_{2} \mathrm{NTs}$ via a template-free approach. The postulated ELMG mechanism for the formation of the $\mathrm{SiO}_{2}$ NTs is illustrated in Figure 1a. The synthesis involves three stages: i) formation of ultrafine, metastable water droplets; ii) collision and merging of the water droplets into linear chains; and iii) shell growth and development of the chains into hollow NTs. The $\mathrm{SiO}_{2} \mathrm{NTs}$ are highly transparent with uniform diameter $(\sim 25 \mathrm{~nm})$, tailored surface properties, tunable shell thickness $(1-5 \mathrm{~nm})$ and high aspect ratio $(\sim 100)$. More importantly, the $\mathrm{SiO}_{2} \mathrm{NTs}$ possess chemically asymmetric surfaces (an organic silane-capped exterior surface and a negatively charged, $-\mathrm{Si}-\mathrm{O}^{-}$-terminated interior surface), which allow the selective loading of positively charged cargoes in the hollow channels through electrostatic interaction. 


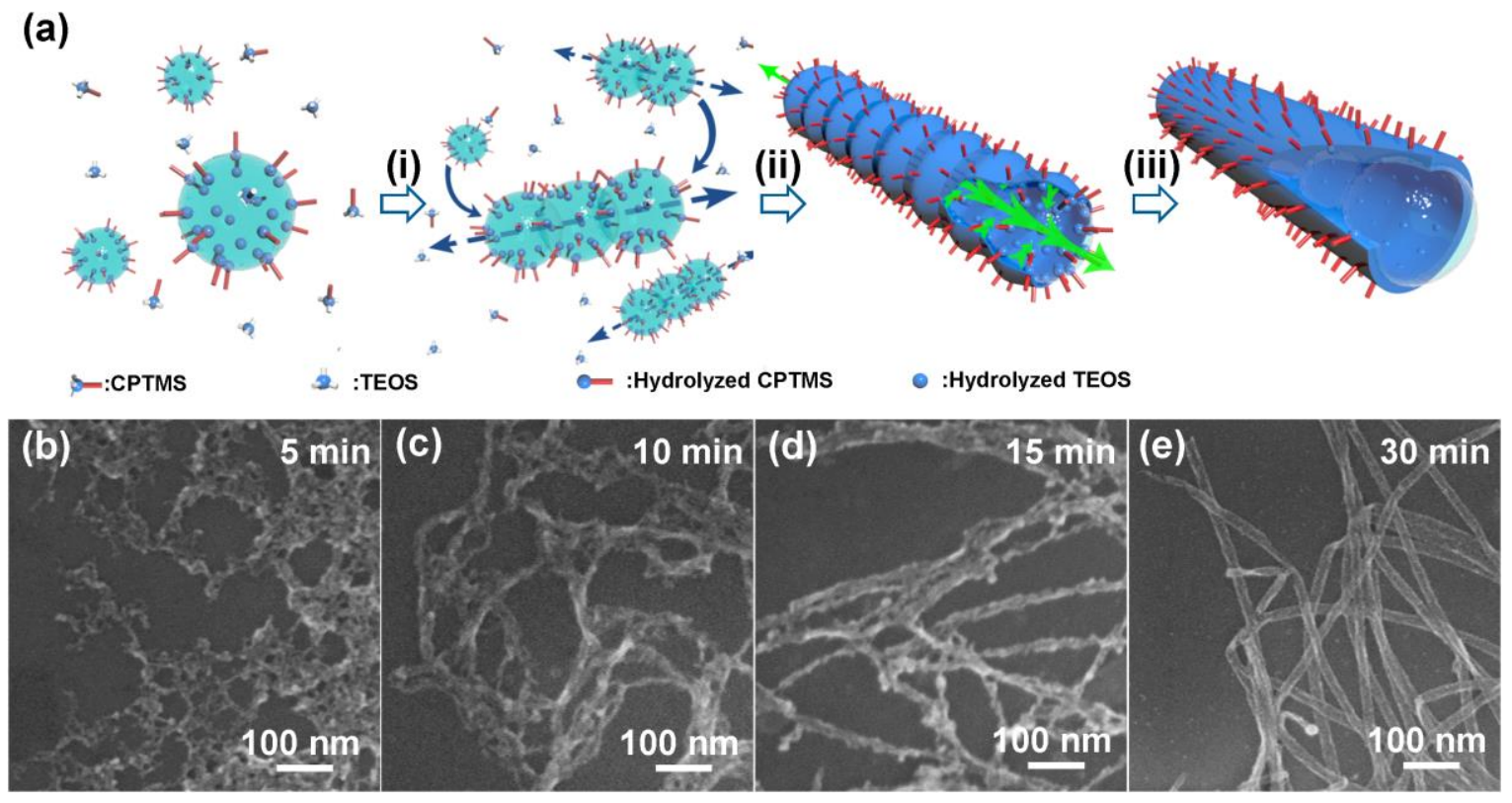

Figure 1. (a) Schematic illustration of the formation of $\mathrm{SiO}_{2} \mathrm{NTs}$ with chemically asymmetric surfaces via an emulsion linear-merging growth approach: (i) collision of ultrafine water nanodroplets; (ii) linear merging of the droplets; and (iii) shell development of the NTs. SEM images of the $\mathrm{SiO}_{2}$ NTs obtained after a growth time of (b) 5, (c) 10, (d) 15 and (e) 30 min. CPTMS, (3chloropropyl)trimethoxysilane; TEOS, tetraethyl orthosilicate.

\section{RESULTS AND DISCUSSION}

The formation mechanism of the $\mathrm{SiO}_{2}$ NTs. A feature of the ELMG approach for silica NTs synthesis is that it affords the formation of ultrafine, metastable water nanodroplets $(\sim 10-20$ $\mathrm{nm})$ that merge into linear chains to reduce the surface energy. The metastable water nanodroplets were prepared through modification of the recently reported water/1-pentanol emulsion system, which has been used for anisotropic growth of high-aspect ratio silica particles from single water droplets with a size of $\sim 100 \mathrm{~nm} .^{24,25,27}$ To obtain the metastable water nanodroplets, distinct changes from the previous formulation were made that include: (i) no addition of polyvinylpyrrolidone as a stabilizer and (ii) the use of ethanol (EtOH) as cosolvent to produce ultrafine water nanodroplets $(\sim 10-20$ $\mathrm{nm}$ ). Owing to the ultrafine size, the emulsified nanodroplets have a high interfacial tension and are thermodynamically metastable. The subsequently added (3- chloropropyl)trimethoxysilane (CPTMS) molecules hydrolyze upon contact with the water droplets under basic conditions. After hydrolysis, CPTMS forms amphiphilic $\mathrm{ClCH}_{2}-\left(\mathrm{CH}_{2}\right)_{2}-\mathrm{Si}(-\mathrm{O}$ )$_{3}$ species, which play a surfactant-like role at the water droplet interface in stabilizing the droplets through insertion of the hydrophobic tail into the 1-pentanol phase while the polar head $\left(-\mathrm{Si}-\mathrm{O}^{-}\right)$faces the water droplets.

The collision of two droplets with soft, amphiphilic $\mathrm{ClCH}_{2}-$ $\left(\mathrm{CH}_{2}\right)_{2}-\mathrm{Si}\left(-\mathrm{O}^{-}\right)_{3}$ arranged at the droplet surface leads to the merging of the droplets into a dumbbell-like shape (Figure 1a). A worm-shaped chain is subsequently obtained with continuous collision and merging of the droplets in a linear direction. Meanwhile, hydrolysis of the added tetraethyl orthosilicate (TEOS), after migrating into the water droplets, and condensation with the hydrolyzed CPTMS at the interface occur to stabilize the shell. Continuous deposition of the hydrolyzed TEOS on the shell from the inner-side of the water droplets extrude the water and produce an elongated nanochannel in center of the chains.

The proposed ELMG mechanism for the NT synthesis is supported by monitoring the shape of the intermediate products through scanning electron microscopy (SEM) (Figure 1be). Worm-shaped particles formed within a time as short as 5 min after addition of TEOS and CPTMS to the nanoemulsion (Figure 1b). Wire structures with grainy, rough surfaces were obtained within 15 min (Figure 1c, d). As the reaction proceeded to $30 \mathrm{~min}$, wires with smooth surfaces were obtained (Figure 1e). These results suggest that the growth of the $\mathrm{SiO}_{2}$ NTs is rapid, which was also confirmed by the dynamic light scattering (DLS) experiments, wherein changes in the particle size as a function of reaction time were monitored (Figure S1).

The shell thickness of the NTs was determined from transmission electron microscopy (TEM) images. The NTs had an ultrathin shell $(\sim 1 \mathrm{~nm})$ after a growth time of $30 \mathrm{~min}$, and showed a partly collapsed, belt-like shape after air-drying (Figure 2a, S2a). The shell thickness increased to $\sim 2 \mathrm{~nm}$ after $1 \mathrm{~h}$ of growth (Figure $2 \mathrm{~b}, \mathrm{~S} 2 \mathrm{~b})$. Prolonged growth $(3 \mathrm{~h})$ led to free-standing NTs with a distinct hollow structure (Figure 2c, $\mathrm{d}, \mathrm{S} 2 \mathrm{c})$. The measured outer diameter, inner diameter and shell thickness of the NTs obtained after a growth time of $3 \mathrm{~h}$ were $\sim 25, \sim 15$ and $\sim 5 \mathrm{~nm}$, respectively (Figure 2c, 2d, S2c). SEM images revealed the size homogeneity of the NTs with length up to several micrometers (Figure 2e, f). Capping of the NTs with a layer of 3-chloropropyl groups was confirmed by the elemental mapping profiles shown in Figure $2 \mathrm{~g}-\mathrm{j}$. Nitrogen sorption analysis revealed that the $\mathrm{SiO}_{2} \mathrm{NTs}$ displayed type IV sorption isotherms, with a hysteresis loop within the relative pressure range of $0.50-0.90$ (Figure $2 \mathrm{k}$ ), indicating the presence of abundant mesopores in the NTs. The NTs had a high surface area of $618 \mathrm{~m}^{2} \cdot \mathrm{g}^{-1}$ (Brunauer-Emmett-Teller method) and pore volume of $1.17 \mathrm{~cm}^{3} \cdot \mathrm{g}^{-1}$. The pore size distribution curve was centered at $\sim 12 \mathrm{~nm}$ (Figure $2 \mathrm{l}$ ), coinciding with the inner

tube 

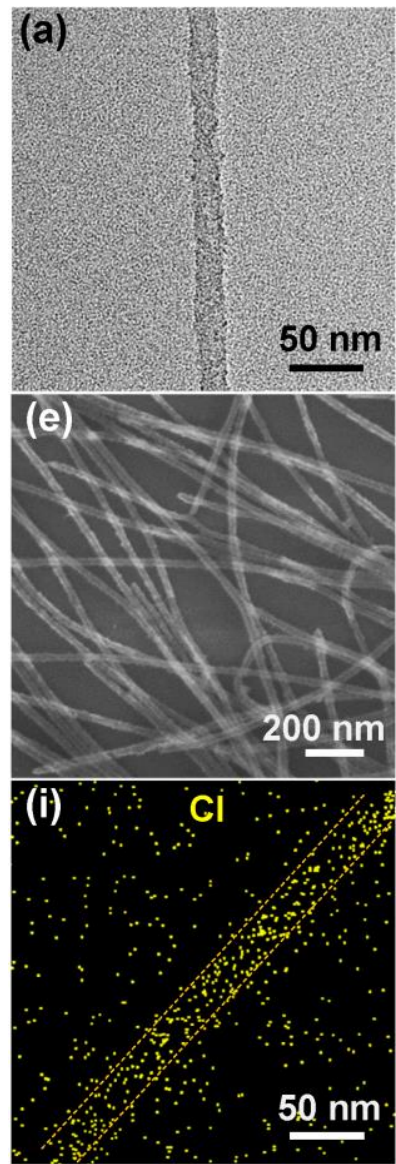
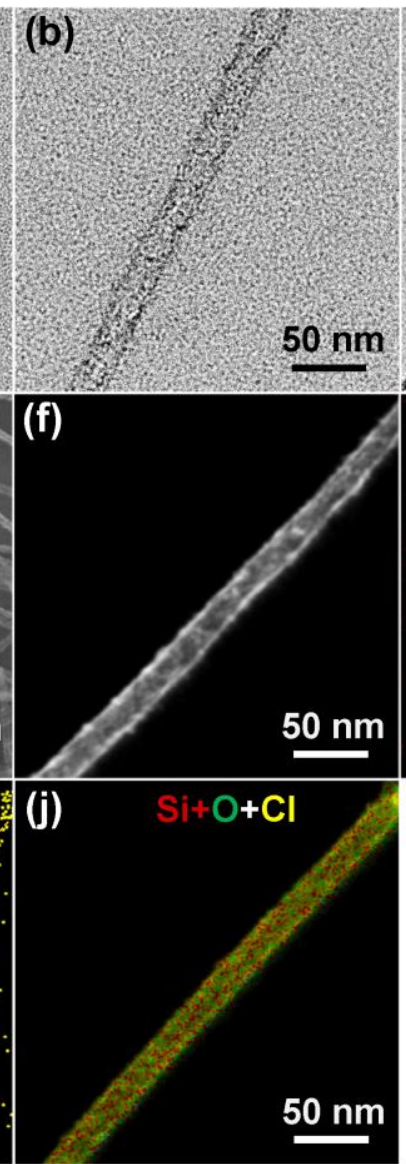

(c)
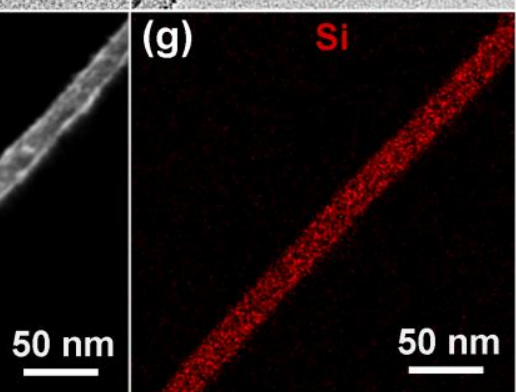

(k)

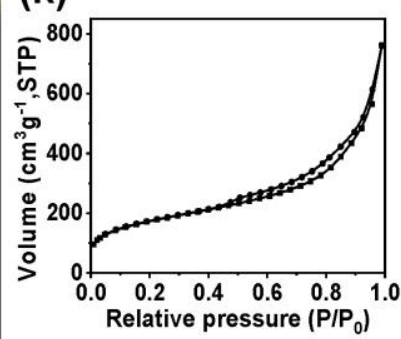

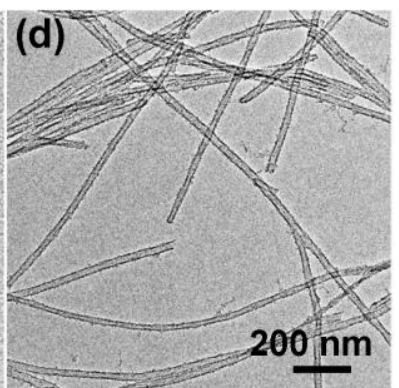

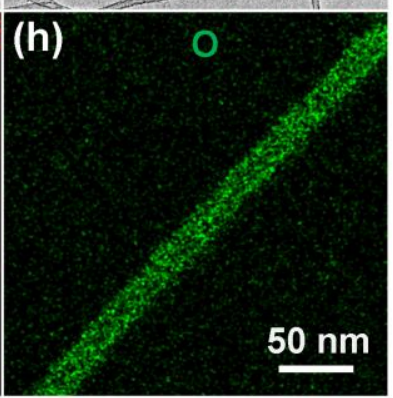

(I)

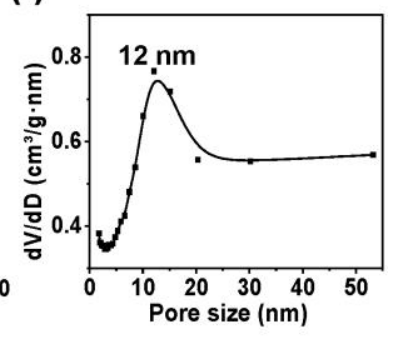

Figure 2. TEM images of the $\mathrm{SiO}_{2}$ NTs obtained at different growth times: (a) 0.5, (b) 1 and (c, d) 3 h. (e, f) SEM images and (g-j) elemental mapping profiles of the $\mathrm{SiO}_{2}$ NTs obtained at a growth time of $3 \mathrm{~h}$. (k) Nitrogen sorption isotherm and (l) pore size distribution of the $\mathrm{SiO}_{2} \mathrm{NTs}$ obtained at a growth time of $3 \mathrm{~h}$.

diameter of $\sim 15 \mathrm{~nm}$ measured from TEM analysis.

We note that the use of $\mathrm{EtOH}$ as a cosolvent is important in the NT synthesis. Without the addition of EtOH, spherical silica particles with a diameter of $\sim 100 \mathrm{~nm}$ were obtained (Figure S3a, b), likely due to the relatively large water droplets formed in the absence of EtOH. When a small amount of $\mathrm{EtOH}(\mathrm{EtOH} / 1$-pentanol, 1:10 v/v) was added to the emulsion system, both $\mathrm{SiO}_{2}$ NTs and a few spherical particles $(\sim 50 \mathrm{~nm})$ formed in solution (Figure S3c, d). Further increases in the $\mathrm{EtOH} / 1$-pentanol ratio over 3:10 resulted in the formation of homogeneous $\mathrm{SiO}_{2} \mathrm{NTs}$, with the outer diameter of the NTs decreasing from 20 to $15 \mathrm{~nm}$ as the EtOH/1-pentanol ratio increased from 3:10 to 1:2 (Figure $\mathrm{S} 3 \mathrm{e}-\mathrm{h}$ ). These results suggest that EtOH plays a key role as cosolvent in controlling the emulsion droplet size - the presence of $\mathrm{EtOH}$ in the water phase would suppress interfacial instabilities, ultimately resulting in the formation of ultrafine water nanodroplets in 1pentanol.

The amount of CPTMS used in the synthesis was also studied. When the CPTMS/TEOS volume ratio was less than 1:20, a mixture of NTs and spherical particles were obtained (Figure S4a-d), likely because the CPTMS amount was insufficient to stabilize the nanoemulsion. When the CPTMS/TEOS ratio increased to $1: 10$, homogeneous NTs were obtained (Figure $\mathrm{S} 4 \mathrm{e}, \mathrm{f})$. A further increase in the CPTMS/TEOS ratio to 1:5 resulted in numerous nanoparticles being deposited on the NT surface (Figure S4g, h).

Optical transparency of the $\mathrm{SiO}_{2}$ NTs. Owing to the ultrathin shell $(<5 \mathrm{~nm})$, suspensions of the $\mathrm{SiO}_{2}$ NTs were almost optically transparent in water. The suspension of $\mathrm{SiO}_{2}$ NTs with a silica concentration of $2.0 \mathrm{mg} \cdot \mathrm{mL}^{-1}$ had a transmittance of $\sim 84 \%$ (at $550 \mathrm{~nm}$ ), which is almost 10 times higher than that of a suspension of $\mathrm{SiO}_{2} \mathrm{NWs}$ with a diameter of $\sim 80$ $\mathrm{nm}$ (Figure 3a). ${ }^{25}$ The flexible and high aspect ratio features of the $\mathrm{SiO}_{2}$ NTs make the assembly of the NTs into a monolithic NT membrane (NTM) through vacuum filtration of the NT suspension possible. The assembled NTM with a thickness of around $200 \mu \mathrm{m}$ (Figure 3e) was highly transparent (Figure 3bd). As observed from Figure 3f, the $\mathrm{SiO}_{2}$ NTs were intertwined into a three-dimensional network and the structure of the NTs was well preserved within the NTM network.

Capping of the $\mathrm{SiO}_{2}$ NTs with other organic groups. Of particular significance is that the ELMG strategy is applicable to the preparation of $\mathrm{SiO}_{2} \mathrm{NTs}$ capped with a variety of organic groups through substituting the CPTMS with another silane group (i.e., phenyltrimethoxysilane, trimethoxysilylpropanethiol, allyltrimethoxysilane) (Figure $4 \mathrm{a}-\mathrm{f}$ ). The phenyl-capped $\mathrm{SiO}_{2}$ NTs can be verified by the FTIR characterization. The silica NTs capped with phenyl groups has skeleton vibrates (C-C) at $1431 \mathrm{~cm}^{-1}$, and bending vibration of $\mathrm{C}-\mathrm{H}$ at $698 \mathrm{~cm}^{-1}$ and $740 \mathrm{~cm}^{-1}$ (Figures S5). UV- 
vis spectra of 3-carboxy-4- nitrophenyl disulfide (DTNB) solution with the addition of thiol-capped silica NTs has a strong absorption at $412 \mathrm{~nm}$,

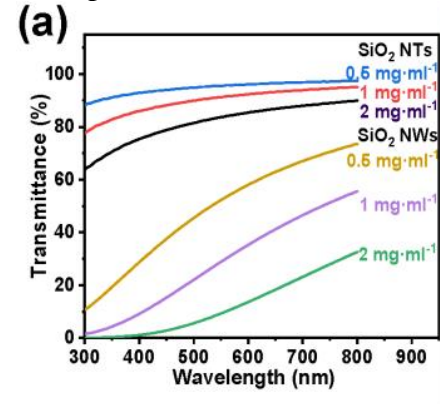

(b)
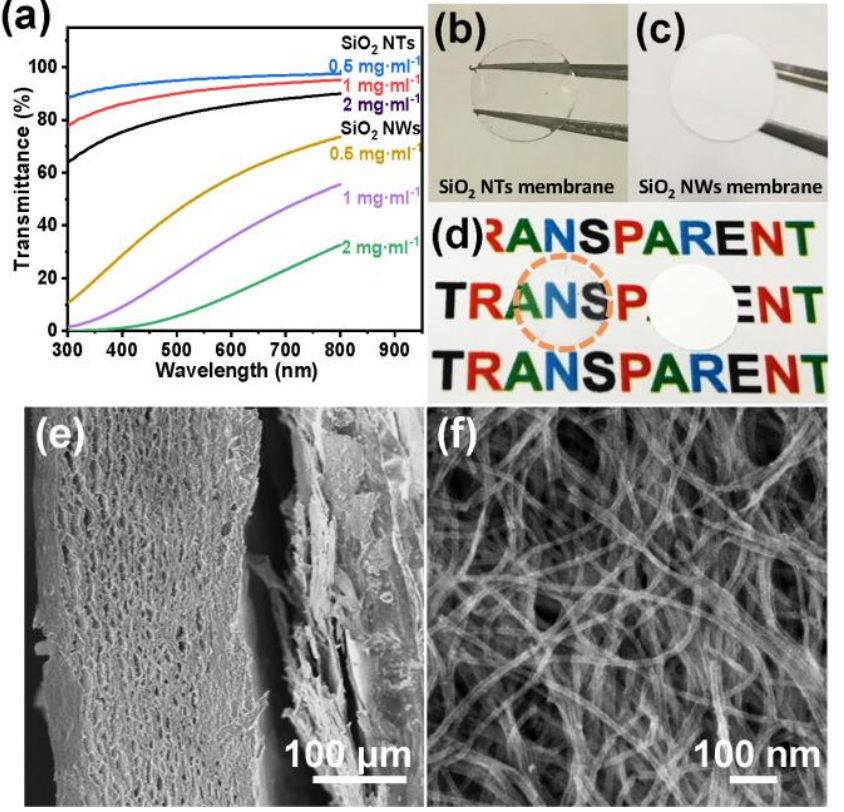

Figure 3. (a) Transmittance spectra of suspensions of $\mathrm{SiO}_{2} \mathrm{NTs}$ and $\mathrm{SiO}_{2} \mathrm{NWs}$ at different silica concentrations. Optical images of a $\mathrm{SiO}_{2}$ NT membrane (NTM) and a $\mathrm{SiO}_{2} \mathrm{NW}$ membrane (NWM) on tweezers (b, c), respectively, and on color-printed paper (d) (NTM is circled on the color-printed paper). Top-view (e) and cross-sectional (f) SEM image of the $\mathrm{SiO}_{2}$ NTM.

indicating that sulfhydryl groups are successful capped on the silica NTs (Figures S6). The amount of capped sulfhydryl silane is calculated to be 10.2 wt.\% based on the weight of silica NTs. When a silane group with a longer hydrophobic chain (e.g., trimethoxy-7-octenyl-silane) was used, particles with a variety of shapes were obtained (Figure S7a, b).

$\mathrm{SiO}_{2}$ NTs for selective cargo loading. The hollow architecture along with the asymmetric surface properties make the $\mathrm{SiO}_{2}$ NTs interesting candidates for selective cargo loading. As a proof-of-principle study, the loading of the NTs with dendritic polyamidoamine (PAMAM) was studied because of the well-defined molecular structure and abundant chemical

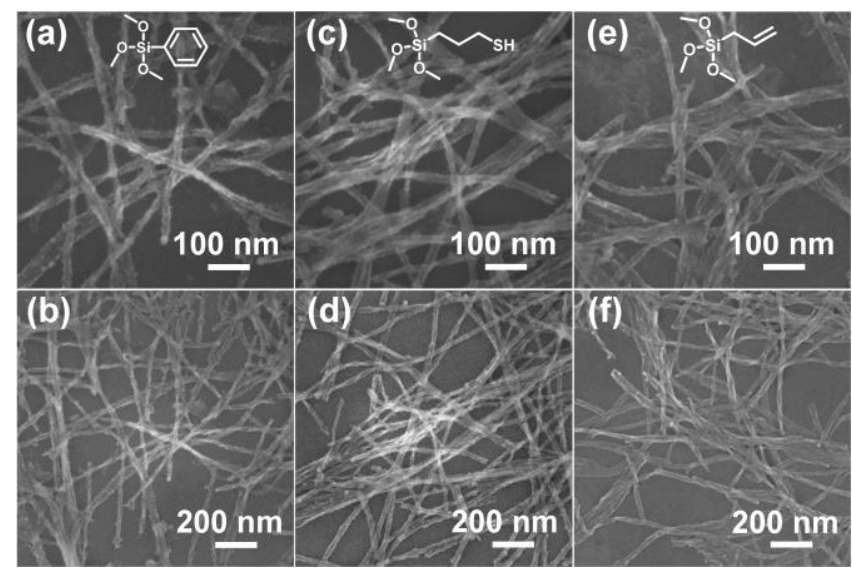

Figure 4. SEM images of the products obtained after replacing the siloxanes (CPTMS) with different silanes: $(a, b)$ phenyltrimethoxysilane; (c,d) trimethoxysilylpropanethiol; (e,f) allyltrimethoxysilane. The reaction time was $1 \mathrm{~h}$ and all other synthesis conditions were the same as described in the 3-chloropropyl groups-capped silica NTs preparation.

groups of this dendrimer for subsequent material functionalization. ${ }^{41-44}$

As illustrated in Figure 5a, amine-terminated PAMAM (denoted as PAMAM- $\mathrm{NH}_{2}$ ) dendrimers impregnate the NTs through electrostatic interaction with the negatively charged ($\left.\mathrm{Si}-\mathrm{O}^{-}\right)$interior surface of the NTs. PAMAM-NH 2 (3.0 G, 3256 $\mathrm{Da}, 2.9 \mathrm{~nm}$ ) could be loaded in the NTs at a loading as high as $63.6 \mathrm{wt} . \%$, as determined by thermogravimetric analysis (Figure S8). Given the excellent metal ion complexing ability, ${ }^{43,44}$ the loaded PAMAM- $\mathrm{NH}_{2}$ dendrimers were then used as a matrix to enrich the interior of the NTs with noble metal ions from the outer solution for in situ reduction of noble metal nanoparticles (NPs) inside the NTs. This "ship-in-a-bottle" strategy for noble metal NP deposition afforded a high loading density of Au NPs with uniform size in the NTs. TEM (Figure $5 \mathrm{~b}, \mathrm{~S} 10 \mathrm{a})$ and elemental mapping (Figure 5c-f) images revealed that the Au NPs (dark dots in Figure 5b, S10a) generated inside the NTs were uniform in size $(\sim 2.5 \mathrm{~nm})$ and evenly distributed inside the NTs. The Au content was 22.4 wt.\%, as determined by inductively coupled plasma atomic absorption spectroscopy (ICP-AAS). In contrast, when the NTs were not pre-loaded with PAMAM- $\mathrm{NH}_{2}$, the formation of $\mathrm{Au} \mathrm{NPs} \mathrm{in}$ the NTs did not occur to an appreciable extent (Figure $5 \mathrm{~g}$ ), thus highlighting the importance of PAMAM- $\mathrm{NH}_{2}$ pre-loading in enriching $\mathrm{Au}(\mathrm{III})$ ions in the NT. Notably, using the same procedure, Au NPs were generated on both the external and internal surfaces of the NTs provided the capped 3chloropropyl groups were removed (via calcination) (Figure $5 \mathrm{~h}$ ). Furthermore, this indicates the importance of the asymmetric surface properties of the $\mathrm{SiO}_{2}$ NTs in achieving selective loading of the NTs. $\mathrm{SiO}_{2}$ NTs loaded with different noble metals $(\mathrm{Pd}, \mathrm{Pt})$ were also prepared, demonstrating the generality of the present approach (Figure S9). TEM images revealed that the Pd NPs and Pt NPs deposited inside the NTs had uniform diameters of about 2 and $1 \mathrm{~nm}$, respectively (Figure S10b, c). The measured contents of Pd and Pt loaded in the NTs were 37.8 and 19.4 wt.\%, respectively, as determined by ICPAAS.

The catalytic activity of the Au NP-loaded NTs was evaluated through the hydrogenation of 4-nitrophenol (4-NP). The reaction was monitored by measuring decreases in the absorbance at $400 \mathrm{~nm}$ using UV-vis spectroscopy (Figure 5i). As deduced from Figure $5 \mathrm{j}$, the reaction could be considered a pseudo-first-order reaction with an apparent rate constant $\left(k_{\text {app }}\right)$ of $0.33 \mathrm{~min}^{-1}$ and a calculated turnover frequency of $2328 \mathrm{~h}^{-1}$. These results indicate that the Au NPs (of which the formation was directed by pre-loading the NTs with PAMAM-NH side the NTs were catalytically active and accessible to the reaction molecules in the bulk solution.

$\mathrm{SiO}_{2}$ NTs for therapeutic agents loading and sustained release. The performance of the chemically asymmetric $\mathrm{SiO}_{2}$ NTs as nanocarriers for therapeutic agents loading was assessed; positively charged doxorubicin (DOX) and lysozyme were examined. DOX is a widely used, small molecule anticancer drug, while lysozyme is often used as a model protein to simulate the in vitro release profile of expensive brainderived neurotrophic factor (BDNF) owing to the similar physical properties (molecular weight, isoelectronic point) that lysozyme and BDNF share. ${ }^{45}$ Dendritic mesoporous silica nanoparticles (DMSNs), ${ }^{40}$ with similar pore size $(\sim 13 \mathrm{~nm})$ and 
pore volume $\left(1.36 \mathrm{~cm}^{3} \cdot \mathrm{g}^{-1}\right)$ to those of the present $\mathrm{SiO}_{2} \mathrm{NTs}$, are used as a control for comparison studies of loading and release performance. Comparison of the performance of
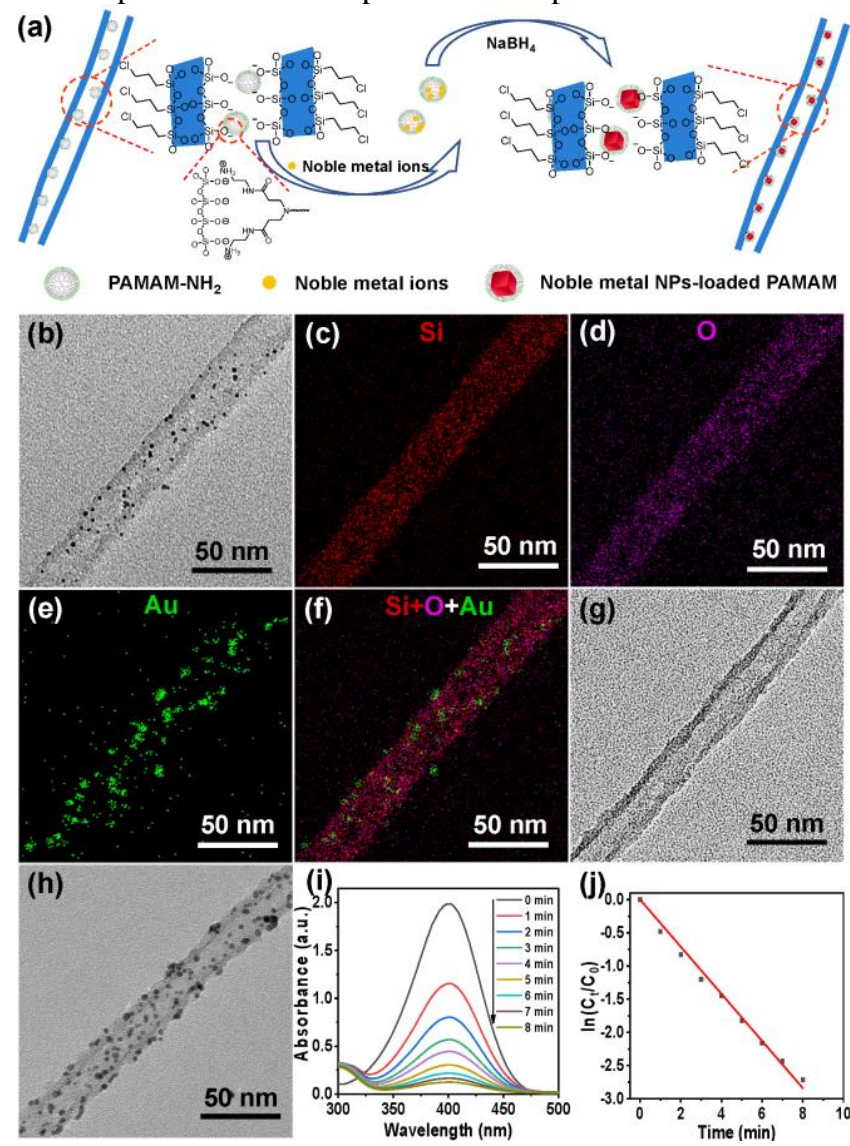

Figure 5. (a) Schematic illustration of amine-terminated polyamidoamine (PMAMA- $\mathrm{NH}_{2}$ ) loading and formation of noble metal nanoparticles inside the $\mathrm{SiO}_{2}$ NTs. The diagrams are not drawn to scale. (b) TEM image and (c-f) element mapping of the $\mathrm{Au}$ NP-loaded $\mathrm{SiO}_{2}$ NTs. (g) TEM image of Au NP-deposited $\mathrm{SiO}_{2}$ NTs without PAMAM-NH 2 pre-loading. (h) TEM image of $\mathrm{Au}$ NP-deposited on the calcined $\mathrm{SiO}_{2}$ NTs. (i) UV-vis absorption spectra of 4-nitrophenol (4-NP) catalytically reduced by Au NPloaded $\mathrm{SiO}_{2} \mathrm{NTs}$ measured at different intervals. (j) Reduction rate profile of 4-NP over the Au NP-loaded $\mathrm{SiO}_{2} \mathrm{NTs}$.

DMSNs and the $\mathrm{SiO}_{2}$ NTs revealed that the latter achieved a high DOX loading of 81.3 wt.\%, which was more than twice that (36.8 wt.\%) achieved in the DMSNs. Similarly, lysozyme loading in the $\mathrm{SiO}_{2}$ NTs (67.6 wt.\%) was significantly higher than that obtained for DMSNs (24.1 wt.\%). These results suggest that electrostatic attraction could be used to efficiently load the $\mathrm{SiO}_{2}$ NTs with a high content of positively charged therapeutics. The high loading capacity of the $\mathrm{SiO}_{2}$ NTs is attributed to the elongated pore structure that suppresses cargo leakage during the washing step, which is undertaken after loading to remove any loosely adsorbed drug molecules on the particle surface.

The in vitro release profiles of the loaded drugs were investigated by incubating the DOX- and lysozyme-loaded carriers in phosphate-buffered saline (PBS, $\mathrm{pH} 7.4$ ) at $37{ }^{\circ} \mathrm{C}$. The amount of DOX and fluorescein isothiocyanate isomer I (FITC)-labeled lysozyme released from the NTs was monitored by fluorescence spectroscopy. The driving force for the release is a concentration gradient of the drug between the nanocarriers and the surrounding PBS medium. DOX and lysozyme release from the DMSNs was rapid, with $>80 \%$ of the loaded cargoes

(a)

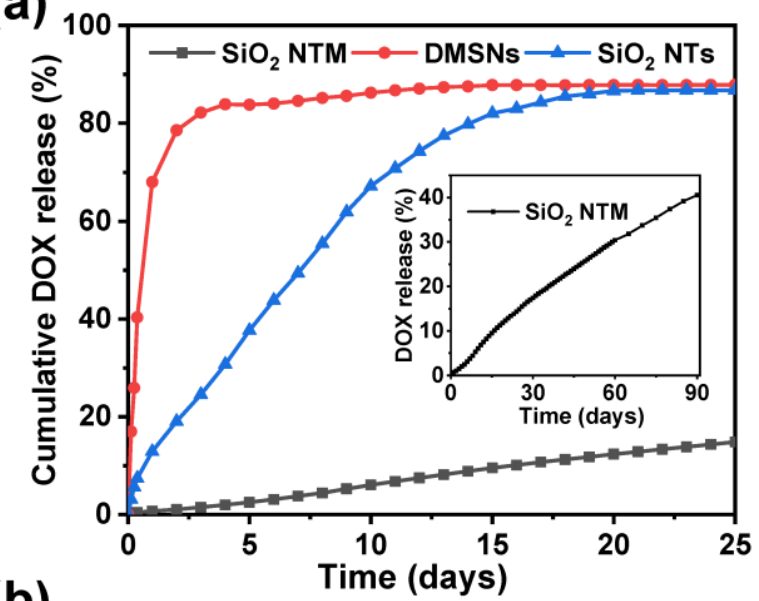

(b)

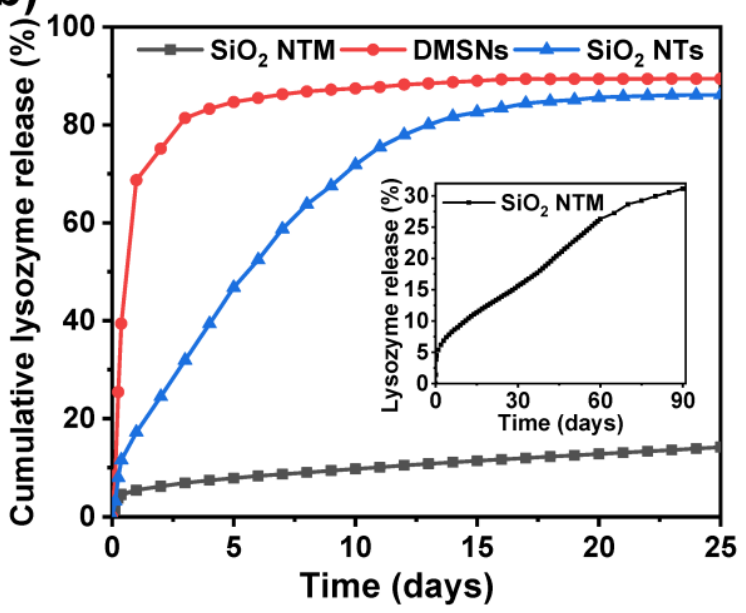

Figure 6. In vitro release profiles of (a) doxorubicin (DOX) and (b) fluorescein isothiocyanate isomer I (FITC)-labeled lysozyme from dendritic mesoporous silica nanoparticles (DMSNs), silica $\mathrm{SiO}_{2} \mathrm{NTs}$ and a silica NT membrane $\left(\mathrm{SiO}_{2} \mathrm{NTM}\right)$.

released within $24 \mathrm{~h}$ (Figure 6). This result was attributed to the radial pore structure of DMSNs, ${ }^{40}$ which affords a relatively short diffusion path $(<100 \mathrm{~nm})$ for the loaded cargoes to diffuse out of the particles. In contrast, cargoes loaded in the $\mathrm{SiO}_{2}$ NTs showed sustained release profiles, with $\sim 80 \%$ of the cargoes released within 2 weeks. The sustained release was attributed mainly to the elongated nanochannel (up to several micrometers) with a narrow diameter $(\sim 15 \mathrm{~nm})$, which could suppress diffusion of the cargoes out of the NTs.

Notably, the release period can be further prolonged through assembling the cargo-loaded $\mathrm{SiO}_{2}$ NTs into a monolithic NTM (diameter: $5.0 \mathrm{~mm}$, thickness: $200 \mu \mathrm{m}$ ). Both DOX and lysozyme release from the NTM were considerably prolonged and showed a near-zero order release profile. Cumulative release amounts of $\sim 40 \%$ for DOX and $\sim 30 \%$ for lysozyme was achieved within a period of 3 months (Figure 6, insets), suggesting the synergistic role of pore structure and geometric size on influencing the release profiles of the $\mathrm{SiO}_{2}$ NTs. These results suggest that the $\mathrm{SiO}_{2}$ NTs and NTM are promising candidates for local drug delivery applications, particularly in where implantation of substance with highlyloaded drugs for achieving a very sustained drug release pro- 
file is interesting ${ }^{14,46,47}$ and interesting for ocular drug delivery owing to the optically transparent properties of the NTs. ${ }^{48,49}$

\section{CONCLUSION}

In conclusion, optically transparent silica NTs with uniform diameter and asymmetrically tunable surface properties were synthesized in the absence of a sacrificial template. Ethanol and CPTMS played key roles in controlling the size and maintaining the metastable state of the water nanodroplets, and subsequently the diameter of the NTs generated. Owing to the asymmetric surface properties of the $\mathrm{SiO}_{2} \mathrm{NTs}$, guest molecules were selectively loaded in the NTs via electrostatic interaction. Using the pre-infiltrated PAMAM- $\mathrm{NH}_{2}$ dendrimer as a matrix, various noble metal nanoparticles were selectively deposited inside the NTs with high density and uniform size. Benefitting from their high specific surface area, large pore volume, and elongated pore structure in the micrometer-scale, $\mathrm{SiO}_{2}$ NTs displayed a high loading capacity for positively charged therapeutic agents and achieved sustained release profiles. The sustained release period could be tuned to last for several months provided the NTs are assembled into a membrane structure. Further studies on the $\mathrm{SiO}_{2} \mathrm{NTs}$ for loading various substances and thus generating multifunctional NTs and NTM for nanocatalysis, sensing and biomedical applications are underway.

\section{EXPERIMENTAL SECTION}

Chemicals. Tetraethyl orthosilicate (TEOS), 1-pentanol, gold(III) chloride trihydrate, fluorescein isothiocyanate isomer I (FITC), (3-chloropropyl)trimethoxysilane (CPTMS), allyltrimethoxysilane and trimethoxy-7-octenyl-silane were purchased from Sigma-Aldrich. Ethanol (EtOH, 99.7\%), ammonia (25 wt.\% solution), sodium borohydride (96\%), and ethylenediaminetetraacetic acid disodium (EDTA-2Na) were obtained from Sinopharm (China). Doxorubicin hydrochloride (DOX), p-nitrophenol (4-NP), trimethoxyphenylsilane and chloroplatinic acid hexahydrate were purchased from Aladdin (Shanghai, China). Polyamidoamine (PAMAM, 2.0 G) with ethylenediamine as core and amine groups as terminal (denoted as PAMAM-NH, 2 wt.\% in ethanol) was obtained from Dendritech Inc. (USA). Lysozyme from hen egg white was purchased from Fluka.

Preparation of Chemically Asymmetric $\mathrm{SiO}_{2} \mathrm{NTs}$. In a typical synthesis, $3.0 \mathrm{~mL} \mathrm{EtOH}$ and $0.42 \mathrm{~mL}$ ammonia aqueous solution were added to $10 \mathrm{~mL} \mathrm{1-pentanol.} \mathrm{The} \mathrm{ammonia} \mathrm{aque-}$ ous solution contained $13 \mathrm{M}$ ammonia and $17 \mathrm{mM}$ EDTA-2Na for catalyzing the hydrolysis and gelation of the silane. After 5 min of vigorously shaking the above solution, $0.1 \mathrm{~mL}$ TEOS and $0.01 \mathrm{~mL}$ CPTMS were added. The mixture was shaken vigorously for $2 \mathrm{~min}$ and then rotated $(60 \mathrm{rpm})$ at room temperature for $3 \mathrm{~h}$. The obtained $\mathrm{SiO}_{2} \mathrm{NTs}$ were centrifuged and washed three times with a water/EtOH mixture (50\%/50\% v/v).

Preparation of Phenyl-, Thiol-, Allyl-, Octenyl-Capped Silica NTs. In a typical synthesis, $3.0 \mathrm{~mL}$ EtOH and $0.42 \mathrm{~mL}$ ammonia aqueous solution (containing $17 \mathrm{mM}$ EDTA-2Na) were added to $10 \mathrm{~mL} 1$-pentanol, and the mixture was vigorously shaken for $5 \mathrm{~min}$. Then, $0.1 \mathrm{~mL}$ TEOS and $0.01 \mathrm{~mL}$ phenyltrimethoxysilane, trimethoxysilylpropanethiol, allyltrimethoxysilane or trimethoxy-7-octenyl-silane were added to the above solution and shaken vigorously for $2 \mathrm{~min}$. The mixture was rotated $(60 \mathrm{rpm})$ at room temperature $3 \mathrm{~h}$. The obtained $\mathrm{SiO}_{2}$ NTs were centrifuged $(8000 \mathrm{~g}, 10 \mathrm{~min})$ and washed three times with a water/EtOH mixture $(50 \% / 50 \% \mathrm{v} / \mathrm{v})$.
Preparation of $\mathrm{SiO}_{2} \mathrm{NT}$ and $\mathrm{SiO}_{2} \mathrm{NW}$ Membrane. $\mathrm{SiO}_{2}$ NWs were synthesized according to our previously reported method. ${ }^{25}$ The vacuum filtration method was used to prepare the free-standing $\mathrm{SiO}_{2} \mathrm{NT}$ and $\mathrm{SiO}_{2} \mathrm{NW}$ membranes. An appropriate amount of diluted NT or NW suspension was vacuum-filtrated through a cellulose acetate filter paper (pore size $0.45 \mu \mathrm{m}$ ) to form free-standing $\mathrm{SiO}_{2} \mathrm{NT}$ and $\mathrm{SiO}_{2} \mathrm{NW}$ membranes, respectively.

Characterization. The formation of the $\mathrm{SiO}_{2}$ NTs was monitored by TEM on an H-600 transmission electron microscope (Hitachi, Japan) and SEM on an Ultra 55 field-emission microscope (Zeiss, Germany). Energy-dispersive X-ray spectroscopy elemental mapping was performed on a Tecnai G2 F20 S-Twin STEM instrument (FEI, USA). Particle size of $\mathrm{SiO}_{2}$ NTs was measured by dynamic light scattering (Zetasizer Nano-ZS (Malvern)). Thermogravimetric analysis of $\mathrm{SiO}_{2} \mathrm{NTs}$ and PAMAM-NH -loaded $\mathrm{SiO}_{2}$ NTs was performed on an SDT Q600-SDT simultaneous thermal analyzer (USA). The organic groups capped on the silica NTs were determined by FT-IR spectroscopy using a Spectrum Two FT-IR spectrometer (PerkinElmer, USA). Nitrogen sorption isotherms were measured on a Micromeritics Tristar 3000 analyzer. The amount of the noble metal (i.e., gold, palladium and platinum) NPs loaded in the $\mathrm{SiO}_{2}$ NTs was determined by inductively coupled plasma atomic adsorption spectrophotometry on an iCAP 7400 spectrophotometer. The amount of DOX or FITClysozyme loaded was monitored by a fluorescence spectroscopy (Shimadzu RF-5301).

\section{ASSOCIATED CONTENT}

\section{Supporting Information}

The Supporting Information is available free of charge on the ACS Publications website.

Experiment detail of synthesis of noble metal NP-loaded $\mathrm{SiO}_{2}$ NTs, catalytic hydrogenation of 4-nitrophenol by Au NP-loaded $\mathrm{SiO}_{2}$ NTs, drug loading and in vitro release, SEM images, HRTEM images of $\mathrm{SiO}_{2}$ NTs and noble metal NP-loaded $\mathrm{SiO}_{2}$ NTs (PDF)

\section{AUTHOR INFORMATION}

\section{Corresponding Author}

*E-mail: yajunwang@ fudan.edu.cn.

\section{ORCID}

Wuli Yang: 0000-0003-0384-9213

Yi Tang: 0000-0002-1463-9927

Frank Caruso: 0000-0002-0197-497X

Yajun Wang: 0000-0002-7557-5883

\section{Notes}

The authors declare no competing financial interest.

\section{ACKNOWLEDGMENT}

This work was financially supported by the National Key R\&D Program of China (2018YFC1602301, 2018YFA0209402), the National Natural Science Foundation of China (21373059), and the Science \& Technology Commission of Shanghai Municipality (16DZ2270100). This work was also supported by the Australian Research Council Centre of Excellence in Convergent Bio-Nano Science and Technology (CE140100036). F.C. acknowledges the award of an NHMRC Senior Principal Research Fellowship 
(APP1135806). Y.W. acknowledges support from the National 1000 Youth Talents Program of China.

\section{REFERENCES}

(1) Margelefsky, E. L.; Zeidan, R. K.; Davis, M. E. Cooperative Catalysis by Silica-Supported Organic Functional Groups. Chem. Soc. Rev. 2008, 37, 1118.

(2) Liang, J.; Liang, Z.; Zou, R.; Zhao, Y. Heterogeneous Catalysis in Zeolites, Mesoporous Silica, and Metal-Organic Frameworks. Adv. Mater. 2017, 29, 1701139

(3) Jin, Q.; Li, X.; Deng, C.; Zhang, Q.; Yi, D.; Wang, X.; Tang, Y.; Wang, Y. Silica Nanowires with Tunable Hydrophobicity for Lipase Immobilization and Biocatalytic Membrane Assembly. J. Colloid Interface Sci. 2018, 531, 555.

(4) Awual, M. R.; Yaita, T.; El-Safty, S. A.; Shiwaku, H.; Suzuki, S.; Okamoto, Y. Copper(II) Ions Capturing from Water Using Ligand Modified a New Type Mesoporous Adsorbent. Chem. Eng. J. 2013, $221,322$.

(5) Wagner, T.; Haffer, S.; Weinberger, C.; Klaus, D.; Tiemann, M. Mesoporous Materials as Gas Sensors. Chem. Soc. Rev. 2013, 42, 4036.

(6) Liu, Y.; Deng, C.; Yi, D.; Wang, X.; Tang, Y.; Wang, Y. Silica Nanowire Assemblies as Three-Dimensional, Optically Transparent Platforms for Constructing Highly Active SERS Substrates. Nanoscale 2017, 9, 15901

(7) Kim, J.; Kim, H. S.; Lee, N.; Kim, T.; Kim, H.; Yu, T.; Song, I. C.; Moon, W. K.; Hyeon, T. Multifunctional Uniform Nanoparticles Composed of a Magnetite Nanocrystal Core and a Mesoporous Silica Shell for Magnetic Resonance and Fluorescence Imaging and for Drug Delivery. Angew. Chem. Int. Ed. 2008, 47, 8438.

(8) Luo, Z.; Cai, K.; Hu, Y.; Zhao, L.; Liu, P.; Duan, L.; Yang, W. Mesoporous Silica Nanoparticles End-Capped with Collagen: RedoxResponsive Nanoreservoirs for Targeted Drug Delivery. Angew. Chem. Int. Ed. 2011, 50, 640.

(9) Yang, P.; Gai, S.; Lin, J. Functionalized Mesoporous Silica Materials for Controlled Drug Delivery. Chem. Soc. Rev. 2012, 41, 3679.

(10) Argyo, C.; Weiss, V.; Braeuchle, C.; Bein, T. Multifunctional Mesoporous Silica Nanoparticles as a Universal Platform for Drug Delivery. Chem. Mater. 2014, 26, 435.

(11) Choi, S.; Choi, Y. j.; Jang, M.-S.; Lee, J. H.; Jeong, J. H.; Kim, J. Supertough Hybrid Hydrogels Consisting of a Polymer DoubleNetwork and Mesoporous Silica Microrods for Mechanically Stimulated on-Demand Drug Delivery. Adv. Funct. Mater. 2017, 27 , 1703826.

(12) Guo, R.; Tian, Y.; Yang, Y.; Jiang, Q.; Wang, Y.; Yang, W. A Yolk-Shell Nanoplatform for Gene-Silencing-Enhanced Photolytic Ablation of Cancer. Adv. Funct. Mater. 2018, 28, 1706398.

(13) Ye, J.; Zhang, H.; Yang, R.; Li, X.; Qi, L. MorphologyControlled Synthesis of $\mathrm{SnO}_{2}$ Nanotubes by Using 1D Silica Mesostructures as Sacrificial Templates and Their Applications in Lithium-Ion Batteries. Small 2010, 6, 296.

(14) Tan, J.; Wang, Y.; Yip, X.; Glynn, F.; Shepherd, R. K.; Caruso, F. Nanoporous Peptide Particles for Encapsulating and Releasing Neurotrophic Factors in an Animal Model of Neurodegeneration. Adv Mater. 2012, 24, 3362

(15) Shimoni, O.; Yan, Y.; Wang, Y.; Caruso, F. Shape-Dependent Cellular Processing of Polyelectrolyte Capsules. Acs Nano 2013, 7, 522

(16) Gu, D.; Schueth, F. Synthesis of Non-Siliceous Mesoporous Oxides. Chem. Soc. Rev. 2014, 43, 313.

(17) Fu, C.; Yi, D. L.; Deng, C.; Wang, X. D.; Zhang, W. J.; Tang, Y.; Caruso, F.; Wang, Y. J. A Partially Graphitic Mesoporous Carbon Membrane with Three Dimensionally Networked Nanotunnels for Ultrasensitive Electrochemical Detection. Chem. Mater. 2017, 29, 5286.

(18) Liu, X.; Zhang, F.; Jing, X.; Pan, M.; Liu, P.; Li, W.; Zhu, B.; Li, J.; Chen, H.; Wang, L.; Lin, J.; Liu, Y.; Zhao, D.; Yan, H.; Fan, C. Complex Silica Composite Nanomaterials Templated with DNA Origami. Nature 2018, 559, 593.
(19) Lin, Y.-S.; Haynes, C. L. Synthesis and Characterization of Biocompatible and Size-Tunable Multifunctional Porous Silica Nanoparticles. Chem. Mater. 2009, 21, 3979.

(20) Wang, S. Ordered Mesoporous Materials for Drug Delivery. Microporous Mesoporous Mater. 2009, 117, 1.

(21) Tang, F.; Li, L.; Chen, D. Mesoporous Silica Nanoparticles: Synthesis, Biocompatibility and Drug Delivery. Adv. Mater. 2012, 24, 1504.

(22) Li, Z.; Barnes, J. C.; Bosoy, A.; Stoddart, J. F.; Zink, J. I Mesoporous Silica Nanoparticles in Biomedical Applications. Chem. Soc. Rev. 2012, 41, 2590.

(23) Kim, J. H.; Hwang, H. J.; Oh, J. S.; Sacanna, S.; Yi, G. R. Monodisperse Magnetic Silica Hexapods. J. Am. Chem. Soc. 2018, 140,9230 .

(24) Kuijk, A.; van Blaaderen, A.; Imhof, A. Synthesis of Monodisperse, Rodlike Silica Colloids with Tunable Aspect Ratio. $J$. Am. Chem. Soc. 2011, 133, 2346.

(25) Yi, D.; Xu, C.; Tang, R.; Zhang, X.; Caruso, F.; Wang, Y. Synthesis of Discrete Alkyl-Silica Hybrid Nanowires and Their Assembly into Nanostructured Superhydrophobic Membranes. Angew. Chem. Int. Ed. 2016, 55, 8375.

(26) Ma, K.; Gong, Y.; Aubert, T.; Turker, M. Z.; Kao, T.; Doerschuk, P. C.; Wiesner, U. Self-Assembly of Highly Symmetrical, Ultrasmall Inorganic Cages Directed by Surfactant Micelles. Nature 2018, 558,577 .

(27) Yi, D. L.; Zhang, Q.; Liu, Y. H.; Song, J. Y.; Tang, Y.; Caruso, F.; Wang, Y. J. Synthesis of Chemically Asymmetric Silica Nanobottles and Their Application for Cargo Loading and as Nanoreactors and Nanomotors. Angew. Chem. Int. Ed. 2016, 55, 14733.

(28) Hu, Y.; Ge, J.; Yin, Y. PDMS Rubber as a Single-Source Precursor for Templated Growth of Silica Nanotubes. Chem. Commun. 2009, 914.

(29) Zhang, M.; Zhang, W.; Wang, S. Synthesis of Well-Defined Silica and Pd/Silica Nanotubes through a Surface Sol-Gel Process on a Self-Assembled Chelate Block Copolymer. J. Phys. Chem. C 2010, 114,15640 .

(30) Gao, C.; Lu, Z.; Yin, Y. Gram-Scale Synthesis of Silica Nanotubes with Controlled Aspect Ratios by Templating of NickelHydrazine Complex Nanorods. Langmuir 2011, 27, 12201.

(31) Zhang, Z.; Shao, C.; Sun, Y.; Mu, J.; Zhang, M.; Zhang, P.; Guo, Z.; Liang, P.; Wang, C.; Liu, Y. Tubular Nanocomposite Catalysts Based on Size-Controlled and Highly Dispersed Silver Nanoparticles Assembled on Electrospun Silicananotubes for Catalytic Reduction of 4-Nitrophenol. J. Mater. Chem. 2012, 22, 1387.

(32) Falk, K.; Sedlmeier, F.; Joly, L.; Netz, R. R.; Bocquet, L. Molecular Origin of Fast Water Transport in Carbon Nanotube Membranes: Superlubricity Versus Curvature Dependent Friction. Nano Lett. 2010, 10, 4067.

(33) Xiong, W.; Du, F.; Liu, Y.; Perez, A., Jr.; Supp, M.; Ramakrishnan, T. S.; Dai, L.; Jiang, L. 3-D Carbon Nanotube Structures Used as High Performance Catalyst for Oxygen Reduction Reaction. J. Am. Chem. Soc. 2010, 132, 15839.

(34) Chen, Z. J.; Guan, Z. H.; Li, M. R.; Yang, Q. H.; Li, C. Enhancement of the Performance of a Platinum Nanocatalyst Confined within Carbon Nanotubes for Asymmetric Hydrogenation. Angew. Chem. Int. Ed. 2011, 50, 4913.

(35) Shi, Z.; Zhang, W.; Zhang, F.; Liu, X.; Wang, D.; Jin, J.; Jiang, L. Ultrafast Separation of Emulsified Oil/Water Mixtures by Ultrathin Free-Standing Single-Walled Carbon Nanotube Network Films. Adv. Mater. 2013, 25, 2422

(36) Zhang, X.; Meng, L.; Lu, Q.; Fei, Z.; Dyson, P. J. Targeted Delivery and Controlled Release of Doxorubicin to Cancer Cells Using Modified Single Wall Carbon Nanotubes. Biomaterials 2009 , 30,6041

(37) Chen, C. C.; Liu, Y. C.; Wu, C. H.; Yeh, C. C.; Su, M. T.; Wu, Y. C. Preparation of Fluorescent Silica Nanotubes and Their Application in Gene Delivery. Adv. Mater. 2005, 17, 404

(38) Seo, Y. D.; Lee, C.; Lee, K. J.; Jang, J. Fabrication of Silica Nanotubes with an Anisotropic Functionality as a Smart Catalyst Supporter. Chem. Commun. 2016, 52, 9825. 
(39) Muellner, M.; Lunkenbein, T.; Breu, J.; Caruso, F.; Mueller, A. H. E. Template-Directed Synthesis of Silica Nanowires and Nanotubes from Cylindrical Core-Shell Polymer Brushes. Chem. Mater. 2012, 24, 1802.

(40) Zhang, K.; Xu, L. L.; Jiang, J. G.; Calin, N.; Lam, K. F.; Zhang, S. J.; Wu, H. H.; Wu, G. D.; Albela, B.; Bonneviot, L.; Wu, P. Facile Large-Scale Synthesis of Monodisperse Mesoporous Silica Nanospheres with Tunable Pore Structure. J. Am. Chem. Soc. 2013, $135,2427$.

(41) Crooks, R. M.; Zhao, M. Q.; Sun, L.; Chechik, V.; Yeung, L. K. Dendrimer-Encapsulated Metal Nanoparticles: Synthesis, Characterization, and Applications to Catalysis. Acc. Chem. Res. 2001, $34,181$.

(42) Wang, C. L.; Zhu, G. S.; Li, J.; Cai, X. H.; Wei, Y. H.; Zhang, D. L.; Qiu, S. L. Rigid Nanoscopic Containers for Highly Dispersed, Stable Metal and Bimetal Nanoparticles with Both Size and Site Control. Chem. - Eur. J. 2005, 11, 4975.

(43) Scott, R. W. J.; Wilson, O. M.; Crooks, R. M. Synthesis, Characterization, and Applications of Dendrimer-Encapsulated Nanoparticles. J. Phys. Chem. B 2005, 109, 692.

(44) Myers, V. S.; Weir, M. G.; Carino, E. V.; Yancey, D. F.; Pande,
S.; Crooks, R. M. Dendrimer-Encapsulated Nanoparticles: New Synthetic and Characterization Methods and Catalytic Applications. Chem. Sci. 2011, 2, 1632.

(45) Mehrotra, S.; Lynam, D.; Maloney, R.; Pawelec, K. M.; Tuszynski, M. H.; Lee, I.; Chan, C.; Sakamoto, J. Time Controlled Protein Release from Layer-by-Layer Assembled Multilayer Functionalized Agarose Hydrogels. Adv. Funct. Mater. 2010, $20,247$.

(46) Wang, Y.; Wise, A. K.; Tan, J.; Maina, J. W.; Shepherd, R. K.; Caruso, F. Mesoporous Silica Supraparticles for Sustained Inner-Ear Drug Delivery. Small 2014, 10, 4244.

(47) Galkina, O. L.; Ivanov, V. K.; Agafonov, A. V.; Seisenbaeva, G. A.; Kessler, V. G. Cellulose Nanofiber-Titania Nanocomposites as Potential Drug Delivery Systems for Dermal Applications. J. Mater. Chem. B 2015, 3, 1688.

(48) Anumolu, S. S.; DeSantis, A. S.; Menjoge, A. R.; Hahn, R. A.; Beloni, J. A.; Gordon, M. K.; Sinko, P. J. Doxycycline Loaded Poly(Ethylene Glycol) Hydrogels for Healing Vesicant-Induced Ocular Wounds. Biomaterials 2010, 31, 964.

(49) Yuan, X.; Marcano, D. C.; Shin, C. S.; Hua, X.; Isenhart, L. C.; Pflugfelder, S. C.; Acharya, G. Ocular Drug Delivery Nanowafer with Enhanced Therapeutic Efficacy. Acs Nano 2015, 9, 1749. 


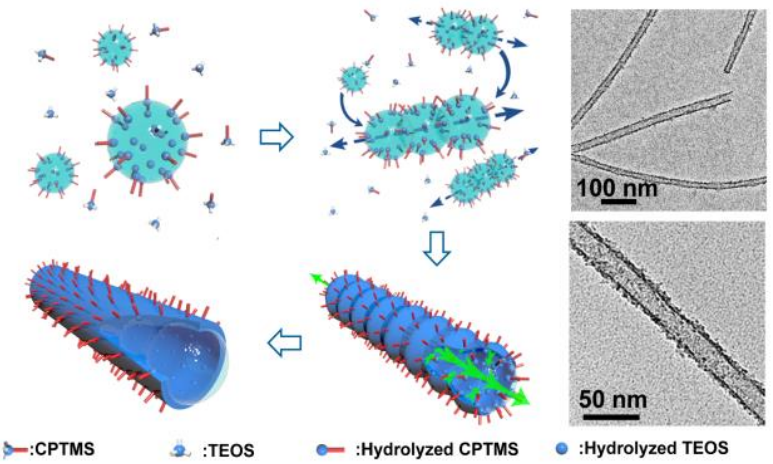

